\title{
Persistent currents and magnetic flux trapping in fragments of carbon deposits containing multiwalled nanotubes
}

\author{
V.I. Tsebro, O.E. Omelyanovskii, \\ P. N. Lebedev Physics Institute, Russian Academy of Sciences, \\ II7924 Moscow, Russia; \\ International Laboratory of High Magnetic Field and Low Temperatures, \\ 53421 Wroclaw, Poland \\ A.P. Moravskii \\ Institute of Chemical Physics, Russian Academy of Sciences, \\ 142432 Chernogolovka, Moscow Region, Russia
}

\begin{abstract}
It is found that the magnetization curves of samples of fragments of cathode carbon deposits with a high content of multiwalled nanotubes exhibit a pronounced irreversible character, attesting to the induction of persistent currents in the samples and to magnetic flux trapping, as happens in a multiply connected superconducting structure. A decrease of the trapped flux in time could not be observed at low (helium) temperatures with a measurement time of about $20 \mathrm{~h}$. For intermediate $(\sim 30 \mathrm{~K})$ and room temperatures the trapped magnetic flux decays slowly with characteristic relaxation times of the order of 150 and $15 \mathrm{~h}$, respectively.
\end{abstract}

PACS 81.05.Ys, 73.23.Ra

1. The electronic properties of carbon nanotubes are the subject of great interest and intensive investigations [1, 2]. Notable among recent works are experimental and theoretical works devoted to coherent electron transport in single-walled nanotubes [3, 4, 5, 6, 7] and theoretical works [8, 9, 10], examining the associated question of circulating, persistent currents in closed toroidal nanotubes.

Transport spectroscopy data [3, 4] show that coherent electron transport occurs in single-walled nanotubes at very low temperatures, and it occurs over very large distances, estimated in Ref.[3] to be right up to the total nanotube lengths of several microns. According to the theoretical results obtained in Ref.[5], the conduction electrons in a single-walled nanotube

*e-mail: tsebro@sci.lebedev.ru 
are affected by the disorder averaged over the circumference of the nanotube, and this results in an increase of the electron mean-free path length with increasing nanotube diameter and, in consequence, exceptional ballistic transport properties over unprecedentedly long distances of the order of $10 \mu \mathrm{m}$ and larger, which is what explains the experimental results. We note that for understandable reasons most theoretical and experimental works on coherent transport concern single-walled nanotubes. For this reason, the result obtained in Ref. [11] is very noteworthy. It was shown there that the conductance of multiwalled carbon nanotubes, ranging in diameter from 5 to $25 \mathrm{~nm}$ and up to $10 \mu \mathrm{m}$ long, measured at room temperature, is quantized in the sense that it does not depend on the nanotube length or diameter, being equal to $G_{0}=2 e^{2} / h=(12,9 k \Omega)^{-1}$. According to Ref.[11], multiwalled carbon nanotubes are capable of carrying at room temperature a current density above $10^{7} \mathrm{~A} / \mathrm{cm}^{2}$, which indicates that high-temperature electric transport in such nanotubes is ballistic and occurs without the release of heat.

On this basis, a very important question is the experimental observation of persistent currents in closed nanotube structures. In the present letter we report the results of measurements of magnetization curves of samples of fragments of cathodic carbon deposits, formed during arc synthesis of multiwalled nanotubes. It follows from the data obtained that the carbon medium of such deposits, which consist of contiguous components with different morphology, the main one being the multiwalled nanotubes, is capable of carrying persistent currents at low (liquid-helium) temperatures or very weakly decaying currents even at high (room) temperatures, and the magnetic flux in such a nanotube is trapped, resulting in hysteresis of the magnetization curves, as happens in a multiply connected superconducting structure.

2. Our experimental samples consisted of small fragments, extracted from the central part of the carbon deposits formed on the cathode during arc synthesis of multiwalled nanotubes in the narrow traditional technology used to fabricate such tubes (see, for example, Ref.[何). Ordinarily, such cathode deposits are subjected to special intense treatment (ultrasonic dispersing followed by treatment with strong oxidizers) in order to remove nanoparticles and other carbon formations and to obtain material consisting of essentially multiwalled nanotubes only. We used for our investigations fragments of deposits that were not subjected, after completion of the arc discharge, to any special treatment that destroys the structure of the material.

The qualitative composition and internal structure of the deposits prepared in different technological regimes, including the samples which we prepared for magnetic measurements, have been investigated in detail in Ref. 12. As a rule, their central part possesses a columnar structure, oriented along the growth axis of the deposit. According to Ref. 12], the carbon 
columns of such a structure consist of three basic components: multiwalled nanotubes ranging in diameter from 5 to $45 \mathrm{~nm}$ (the most likely value is $\sim 15 \mathrm{~nm}$ ), multiwalled polyhedral particles ranging in size from 20 to $90 \mathrm{~nm}$, and curved graphitized formations. Their relative amounts and characteristic sizes are determined by the parameters of the arc process. While all three components are present inside the columns, the outer shell of the columns consists predominantly of only intertwined multiwalled nanotubes. Multiwalled nanotubes in the form of chaotic braids are also present in the space between the columns. Nanotubes from different parts of a deposit are oriented predominantly at obtuse angles with respect to its growth axis.

The samples used for the measurements of the magnetization curves consisted of either (1) carbon columns (average column diameter $\sim 50 \mu \mathrm{m}$ ) obtained from the center of the deposit, assembled and held together with a negligible amount of nonmagnetic glue, and oriented along the $z_{c}$ axis or (2) small, $\sim 2.5 \mathrm{~mm}$ in diameter, bulk cylinders cut from the center of the deposit along the growth axis $z_{d}$. In the first variant the samples ranged from one to several milligrams in mass, and they were used for measurements of the magnetization curves in weak magnetic fields $(<500$ Oe) using a SQUID magnetometer with a sensitivity of the order of $5 \cdot 10^{-9}$ emu with respect to the magnetic moment. Figure 1 shows an optical image of the profile of the end of a sample (No. 196-1s), consisting of carbon columns assembled together. In the second variant the cylindrical samples were of the order of several tens of milligrams in mass, and they were used for magnetic measurements in strong magnetic fields using a self-compensated magnetometer with a capacitance sensor [13].

3. Weak magnetic fields. Figure 2 shows the results of measurements performed on a SQUID magnetometer at $T=4.2 \mathrm{~K}$ of the magnetization curve of sample No. 196-1s (sample mass $1.65 \mathrm{mg}, H \perp z_{c}$ ), held for a long time at room temperature in a zero (the Earth's) magnetic field. The initial increase of the field in this case always results in a virtually linear dependence $M(H)$ (curve 1) with slope (magnetic susceptibility) for this sample equal to $\chi=-3.8 \cdot 10^{-4} \mathrm{emu} /($ mole $\mathrm{C})$. As the magnetic field decreases (curve 2), trapped magnetic flux, corresponding in this case to a paramagnetic moment $M_{r} \approx 0.04 \mathrm{emu} /($ mole $\mathrm{C})$, remains in the sample, and with further cycling of the magnetic field from -500 to +500 Oe (curves 2 and 3) a characteristic hysteresis loop is observed.

Long-time (up to $20 \mathrm{~h}$ ) observations of $M_{r}$, at liquid-helium temperature did not show, within the limits of measurement accuracy $(\sim 1 \%)$, any appreciable decrease of the moment; this indicates that the currents induced in the sample are persistent at low (liquid-helium) temperatures. Measurements of $M_{r}$ is a function of time and temperature at higher temperatures showed that up to room temperatures $M_{r}$ depends mainly on the time and not the temperature. Thus, when the sample is heated to intermediate temperatures $(\sim 20 \mathrm{~K}), M_{r}$ does not change but it already shows appreciable, exponen- 
tial, relaxation with characteristic relaxation time $\tau_{0} \sim 150 \mathrm{~h}$. When the sample is heated relatively rapidly (in order to eliminate the time factor) up to room temperature, $M_{r}$ decreases by several percent, and measurements of the time dependencies $M_{r}(t)$ showed that for such high temperatures the relaxation time $\tau_{0}$ remains quite long, of the order of $15 \mathrm{~h}$. Therefore, in order to return the sample essentially into the initial state (which means, for example, $M_{r}$ is decreased to a level $<1 \%$ of the initial value), the sample must be held at room temperature for approximately three days.

A repeated check of different samples prepared from different parts of the same deposit and from different deposits showed that hysteresis of the magnetization curves occurs in virtually all cases - only its magnitude changes, and in very wide limits from sample to sample. For a number of samples the contribution of the irreversible part of the magnetization was very small, and the hysteresis properties of the $M(H)$ curves could be illustrated satisfactorily only by presenting the difference curves $M(H)$ $\chi_{0} H$ (where $\chi_{0}$ is the static magnetic susceptibility at the extreme points of the hysteresis loop). In this sense the data presented in Fig. 2 are better, in terms of the magnitude of the effect, than the data that we obtained in weak magnetic fields for samples consisting of carbon columns.

We also note that the magnitude of the hysteresis of the magnetization curves of samples consisting of carbon columns depends very strongly on the direction of the magnetic field relative to the axis $z_{c}$ of the columns. The magnetic susceptibility itself or the magnetization of the sample is also anisotropic. But the magnetization anisotropy in weak magnetic fields is very small - of the order of several percent - and the magnetic susceptibility is, as a rule, larger for $H \| z_{c}$, whereas the hysteresis of the $M(H)$ curves changes severalfold as a function of the orientation of the sample, the effect being maximum for $H \perp z_{c}$. As an example, Fig. 3 shows for one of the samples (No. 196-11) the complete hysteresis loop of the form $M(H)-\chi_{0} H$ for $H \perp z_{c}$ and the initial curves $M(H)-\chi_{0} H$ with increasing and decreasing magnetic field with the orientation $H \| z_{c}$. It is evident that the trapped flux $M_{r}$ for $H \| z_{c}$ is approximately four times smaller than for $H \perp z_{c}$.

4. Strong magnetic fields. The measurements were performed at $T=$ $4.2 \mathrm{~K}$ in the nonuniform field of a superconducting solenoid using a selfbalancing magnetometer with a capacitance sensor [13] on cylindrical samples cut from the central part of the deposit along the growth axis $z_{d}$ of the deposit. The magnetic field was not switched, i.e., the $M(H)$ curves were measured with increasing and decreasing fields. Figure 1 shows magnetization curves for one of the samples (No. 140) with increasing and decreasing magnetic field for two orientations: $H \| z_{d}$ (curve 1) and $H \perp z_{d}$ (curve 2). These data illustrate the general pattern of the results obtained in strong magnetic fields. In the first place, the magnetization curves are strongly nonlinear, which indicates the complicated character of the magnetic in- 
teractions in the system. We note that in the process there is a strongly nonlinear field dependence of the magnetization anisotropy, where rapid growth of the ratio $M_{\|} / M_{\perp}$ in the field range $0-20 \mathrm{kOe}$ is replaced by slow monotonic growth of the ratio up to $M_{\|} / M_{\perp} \sim 1.5$ for $H \sim 100 \mathrm{kOe}$. In the second place, the magnetization curves are irreversible even in strong magnetic fields. Just as in weak magnetic fields and for carbon column samples, the hysteresis of the $M(H)$ curves is appreciably anisotropic, but the hysteresis is greater for $H \| z_{d}$ (see Fig. 团). We also note that when the field completely leaves the region of strong magnetic fields, for some samples the residual moment $M_{r}$ reaches values $\sim 2 \mathrm{emu} /\left(\right.$ mole $\mathrm{C}$ ) for $H \| z_{d}$.

5. In summary, we have observed that samples of fragments of cathode carbon deposits, which were not damaged by special treatment in order to remove the multiwalled nanotubes contained in them, can carry persistent magnetic-field induced currents at low temperatures (liquid-helium) or very weakly decaying currents at high (room) temperatures. This property is observed for magnetization curves that show a pronounced irreversible character, i.e., magnetic flux is trapped in the samples, as happens in a multiply connected superconducting structure.

At present one can only surmise how the system of paths conducting persistent or weakly decaying currents in the carbon medium of such samples is organized. It is possible that the structure is similar to a so-called "Mendelssohn sponge" [14] (a multiply connected system of thin superconductor strands in a normal matrix), and the character of the irreversible behavior of its magnetization corresponds to the well-known critical-state model [15]. If this is so, then the virtually linear dependence $M(H)$ with the field increasing initially (see curve 1 in Fig. 2) indicates that the critical current of the filaments is very high, and the corresponding field-dependent penetration depth of the magnetic field in such a sponge is small. What comprises such a sponge structure is also unclear, but in this case there is a system of interconnected nanotubes in which electric transport is loss-free or the losses are negligibly small. According to electron-microscope data [12], the structure of the outer shell of the carbon columns of the deposit matches such a sponge structure; such a shell consists of a quite dense network of intertwined and interconnected nanotubes. Since the planes of the cells of such a network are perpendicular to the axis of the columns, the large anisotropy of the hysteresis of the magnetization curves and the fact that the trapped flux is greater in the case $H \perp z_{c}$ become understandable. A more sparse but also micron-size network of nanotubes is also present in the space between the columns along their entire length [12]. This reticular structure, where the planes of the cells are perpendicular to the growth axis of the deposit, likewise seems to trap magnetic flux well, and its effect becomes determining in bulk samples cut from a deposit, the trapped flux being maximum for $H \| z_{d}$. Since, as noted above, the hysteresis of the $M(H)$ curves varies very strongly from sample to sample, it can be inferred that it is the quality of 
the intertube connections that determines the wholeness of the sponge and the corresponding trapping of the magnetic flux, leading to hysteresis of the magnetization curves. As a rule, appreciable hysteresis of the magnetization curves has always been observed in samples whose magnetic susceptibility at low temperatures was much greater in absolute magnitude than the value adopted for nanotubes $\chi \sim-3 \cdot 10^{-4} \mathrm{emu} /($ mole C) [16, 17]. Apparently, persistent currents already make a large contribution to the diamagnetic response of such samples to an external magnetic field. It has not been ruled out that the proposed current-carrying sponge does not work in the manner that has been supposed, as a whole, in which case it must be assumed that magnetic flux is trapped in individual cells of the nanotube network which are unconnected or weakly connected with one another. Further investigations are required to clarify the nature of the observed persistent currents and the corresponding current-carrying structure.

We thank the State Scientific and Technical Program "Topical Problems in Condensed-Matter Physics" for support ("Fullerenes and Atomic Clusters" No. 2-5-99).

\section{References}

[1] T. W. Ebbesen, Physics Today 49, 26 (1996).

[2] R. E. Smalley, Rev. Mod. Phys. 69, 723 (1997).

[3] S. J. Tans, M. H. Devoret, H. Dai, A. Thess, R. E. Smalley, L. J. Geerligs, and C. Dekker, Nature 386, 474 (1997).

[4] M. Bockrath, D. H. Cobden, P. L. McEuen, N. G. Chopra, A. Zettl, A. Thess, and R. E. Smalley, Science 275, 1922 (1997).

[5] C. T. White and T. N. Todorov, Nature 393, 240 (1998).

[6] R. Egger and A. O. Gogolin, preprint cond-mat/9803128.

[7] M. Bockrath, D. H. Cobden, J. Lu, A. G. Rinzler, R. E. Smalley, L. Balents, and P. L. McEuen, Nature 397, 598 (1999).

[8] R. C. Haddon, Nature 388, 31 (1997).

[9] M. F. Lin and D. S. Chuu, Phys. Rev. B 57, 6731 (1998).

[10] A. A. Odintsov, W. Smit, and H. Yoshioka, preprint cond-mat/9805164.

[11] S. Frank, P. Poncharal, Z. L. Wang, and W. A. de Heer, Science 280, 1744 (1998).

[12] N. A. Kiselev, A. P. Moravsky, A. B. Ormont, and D. N. Zakharov, Carbon 37, 1093 (1999). 
[13] Null-balance magnetometer with capacitance sensor, internal specification, International Laboratory of High Magnetic Fields and Low Temperatures, Wroclaw, Poland, 1998.

[14] K. Mendelssohn, Proc. Roy. Soc. A152, 34 (1935).

[15] C. P. Bean, Phys. Rev. Lett. 8, 250 (1962).

[16] R. C. Haddon, Nature 378, 249 (1995).

[17] M. S. Dresselhaus, G. Dresselhaus, and P. C. Eklund, Science of Fullerenes and Carbon Nanotubes (Academic Press, Inc., San Diego, California, 1996), p. 836. 
Figure 1: Optical image of the profile of the end of sample No. 196-1s, consisting of carbon columns assembled together. 


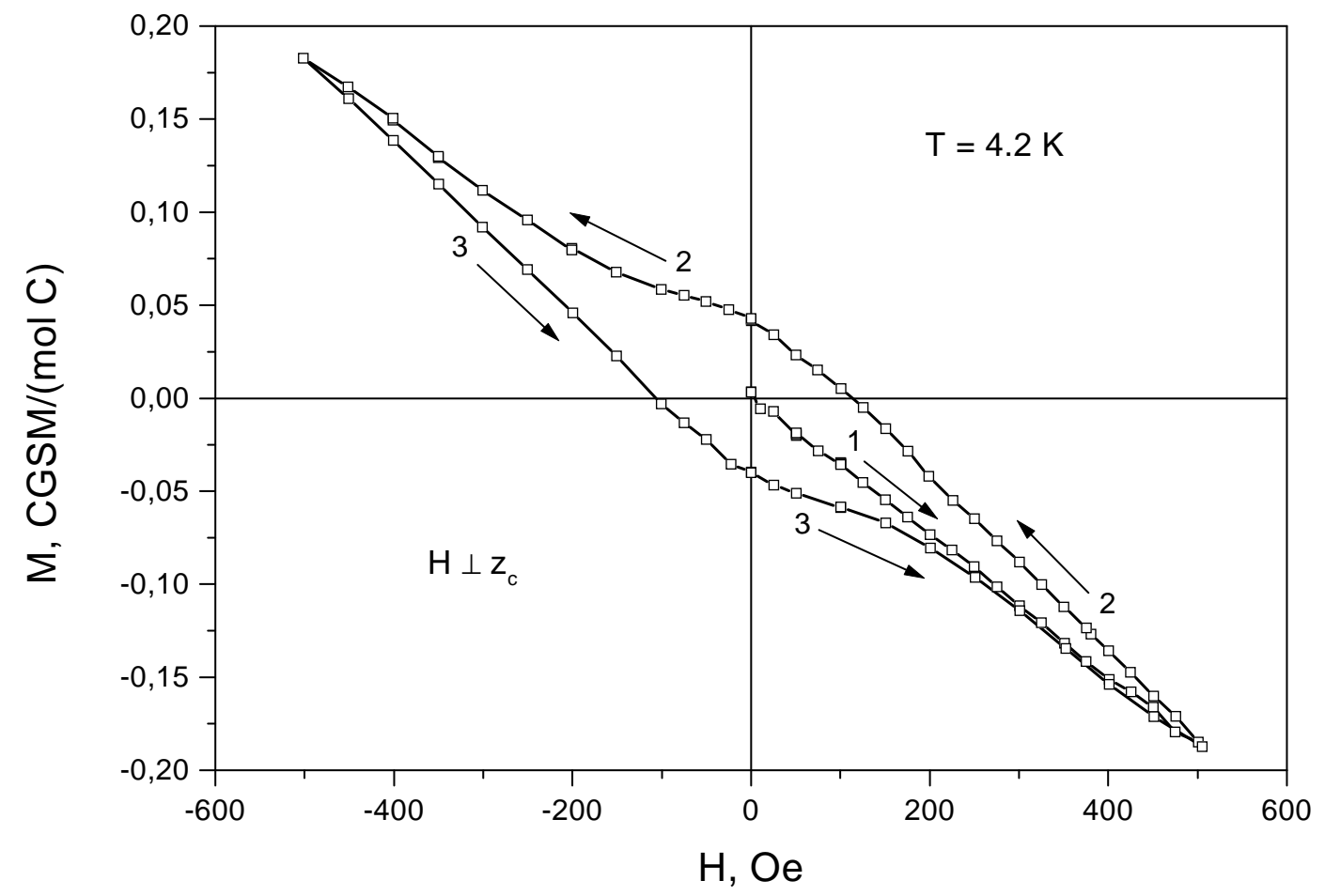

Figure 2: Hysteresis loop of the magnetization curve of sample No. 196-1s at $T=4.2 \mathrm{~K}$. The sample mass is $1.65 \mathrm{mg}$. The magnetic field is directed perpendicular to the axis of the carbon columns. 


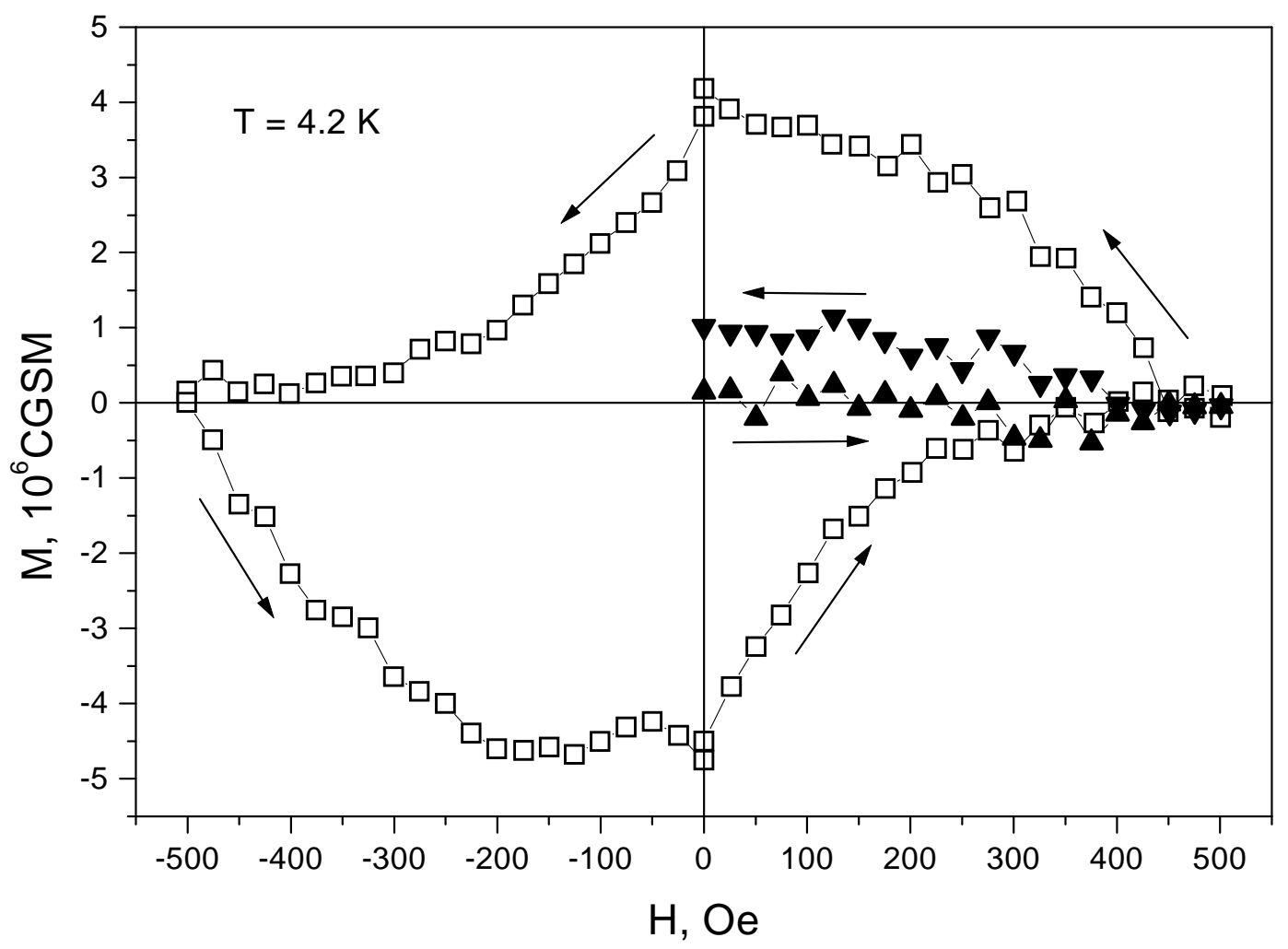

Figure 3: Hysteresis anisotropy of the magnetization curves for sample No. $196-11$ at $T=4.2 \mathrm{~K}$. The absolute values of the magnetic moment minus $\chi_{0} H$ are presented (see text): $\square$ - complete hysteresis loop for $H \perp z_{c}$, initially increasing $(\boldsymbol{\Lambda})$ and decreasing $(\boldsymbol{\nabla})$ magnetic field for $H \| z_{c}$. 


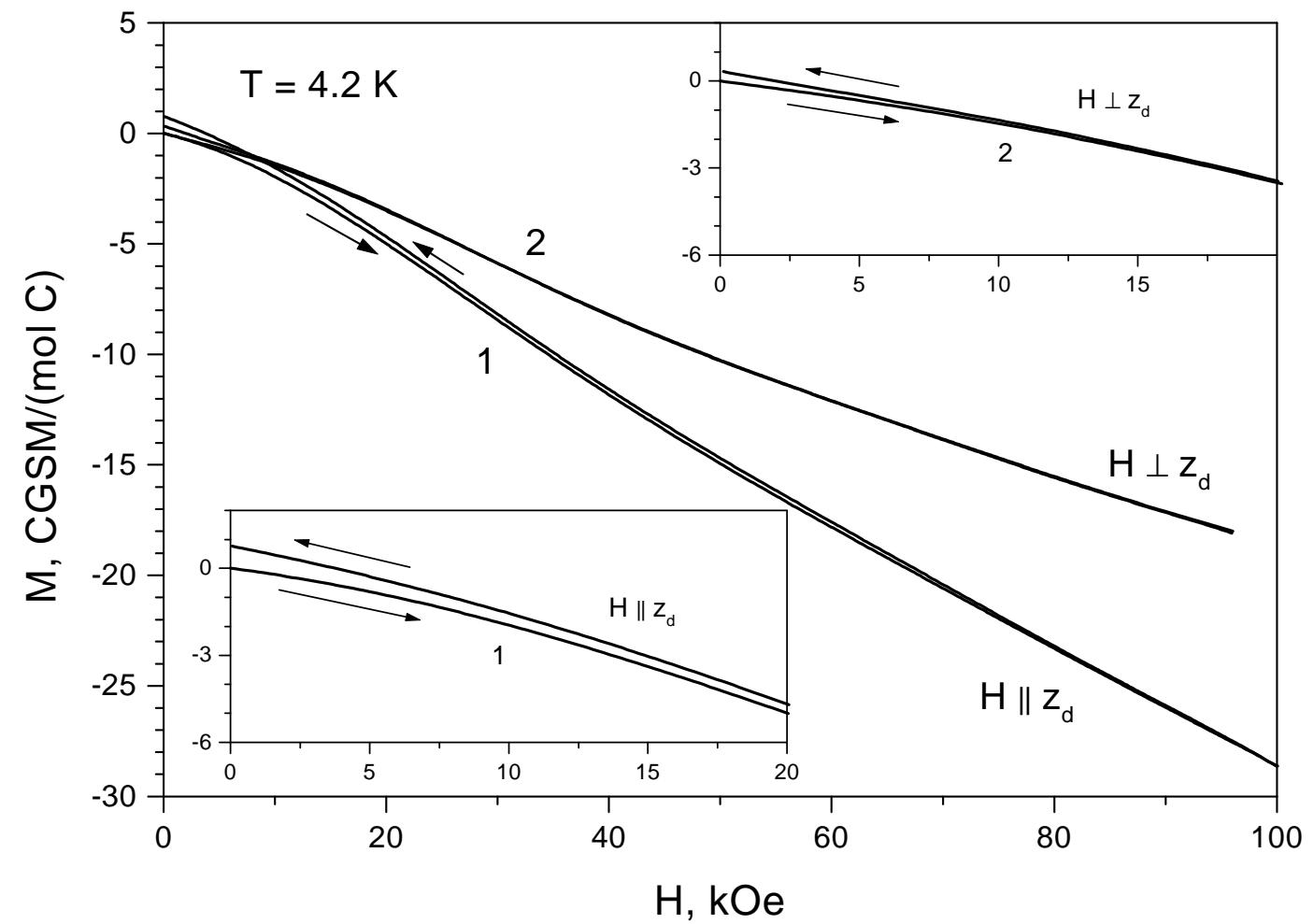

Figure 4: Magnetization curves for sample No. 140 in strong, increasing and decreasing magnetic fields with orientation along (1) and perpendicular (2) to the growth axis of the deposit. $T=4.2 \mathrm{~K}$. The sample mass is 66.6 mg. Inserts: Initial sections of the curves for different orientations of the magnetic field. 
This figure "fig1.jpg" is available in "jpg" format from: http://arxiv.org/ps/cond-mat/9911432v1 